\title{
Simulação do Processo de Injeção de Polipropileno Isotático (iPP) Utilizando um Modelo de Cinética de Cristalização Quiescente.
}

\author{
Marcos A. d'Ávila, Carlos H. Ahrens e Rosario E. S. Bretas
}

Resumo: Este trabalho concentrou-se na simulação das fases de preenchimento e resfriamento do processo de injeção do polipropileno isotático. Foi utilizado um modelo matemático baseado nas equações de conservação onde foi considerada a cinética de cristalização quiescente como termo fonte na equação da energia. Os parâmetros do modelo de cinética de cristalização, assim como os do modelo de viscosidade, foram obtidos experimentalmente. Para a solução das equações governantes foi utilizado o método dos volumes finitos. Como resultados foram obtidos os campos de temperatura, pressão, velocidade, taxa de cisalhamento e cristalinidade em diferentes condições de processamento.

Palavras-chave: Moldagem por injeção, simulação, método dos volumes finitos, cinética de cristalização, polipropileno.

\section{Introdução}

Atualmente o processo de moldagem por injeção mostra-se como um dos principais processos de transformação de polímeros devido à sua grande versatilidade e consequentemente, grande aplicabilidade. Quando um polímero é processado, suas propriedades finais são fortemente influenciadas pelas condições de processamento ${ }^{[1]}$. Assim, é necessário estudar o processo de injeção a partir do modelamento matemático do processo utilizando equações baseadas nos princípios de conservação, que é fundamental para compreender os efeitos do processamento nas propriedades finais da peça moldada. No caso do projeto de moldes de injeção, o domínio do processo mostra-se de grande importância devido ao alto custo dos moldes de injeção, onde uma falha no projeto pode acarretar em um aumento excessivo no custo final de fabricação do molde ${ }^{[2]}$.

Basicamente, o processo de injeção consiste em forçar o polímero para dentro de uma cavidade após este ter sido fundido e homogeneizado. Neste processo ocorrem três fases: preenchimento, empacotamento e resfriamento. A fase de preenchimento ocorre quando o material, devido à aplicação de pressão, escoa dentro da cavidade até preenchê-la. A fase de empacotamento consiste na adição de mais material na cavidade para compensar o encolhimento que ocorre devido ao resfriamento da massa dentro da cavidade. O resfriamento se dá desde o início do preenchimento até a abertura do molde.

A forma como o polímero resfria dentro da cavidade é muito importante devido aos efeitos de orientação molecular na peça e preenchimento do molde.

Marcos A. d'Ávila, Carlos H. Ahrens - Departamento de Engenharia Mecânica - Universidade Federal de Santa Catarina; Rosario E. S. Bretas - Departamento de Engenharia de Materiais - Universidade Federal de São Carlos 
Para polímeros semicristalinos essa fase é essencial devido ao processo de cristalização que se dá de forma não isotérmica, ocasionando diferentes taxas de cristalização para cada posição na peça ${ }^{[3]}$. O desenvolvimento de cristais de morfologias diferentes ao longo da espessura da peça, irá influenciar as propriedades finais da peça injetada, como pode-se observar nas referências 4 e 5 . Portanto, no estudo do processo de injeção de polímeros semicristalinos é de fundamental importância considerar a cinética de cristalização.

São poucos os trabalhos de simulação do processo de injeção de polímeros semicristalinos. Lafleur e Kamal ${ }^{[6,7]}$ desenvolveram um modelo capaz de prever propriedades finais da peça injetada, como o módulo elástico e a espessura da camada solidificada, levando em consideração a viscoelasticidade e a cinética de cristalização quiescente. $\mathrm{O}$ método numérico utilizado para solução das equações governantes foi o método das diferenças finitas.

Brito $^{[8,9]}$ propôs um método para prever, pela simulação numérica utilizando o método das diferenças finitas, a camada superficial "skin layer" baseando-se no conceito da temperatura de não fluxo (temperatura na qual o polímero cessa o seu escoamento) em peças injetadas de polipropileno, porém não foi utilizado um modelo de cinética de cristalização.

Hsiung e Cakmak ${ }^{[10]}$ fizeram um estudo de simulação das fases de preenchimento e resfriamento para determinar gradientes de cristalinidade desenvolvidos em polímeros de cinética de cristalização lenta pelo método das diferenças finitas. Foi considerado o modelo de Nakamura de cristalização não isotérmica ${ }^{[11]}$ onde os parâmetros deste modelo foram considerados como dependentes da tensão através de uma relação empírica. Neste trabalho, os gradientes de cristalinidade obtidos na simulação concordaram qualitativamente com os resultados experimentais.

Isayev et $\mathrm{a}^{[12,13]}$ desenvolveram um estudo do processo de injeção de polímeros semicristalinos considerando o preenchimento e o empacotamento, utilizando o método das diferenças finitas. O modelo de cinética de cristalização quiescente utilizado foi o de Nakamura ${ }^{[10]}$, onde foi utilizada a teoria de HoffmanLauritzen $^{[14]}$ para determinar o parâmetro de taxa do modelo, seguindo o procedimento feito por Chan e Isayev $^{[15]}$. Neste trabalho foi também considerada a cristalização induzida por fluxo utilizando o modelo de Janeschitz-Krieg ${ }^{[16,17]}$ na simulação. Com esse modelo os autores foram capazes de predizer a transição da camada superficial orientada com o centro cristalizado de forma quiescente. Com os resultados da simulação sobre o desenvolvimento da cristalinidade foi possível observar as diferentes cinéticas de cristalização que ocorrem de forma quiescente e induzida por fluxo.

No presente trabalho foi dada atenção na simulação das fases de preenchimento e resfriamento do processo de injeção de um polímero semicristalino, onde os parâmetros dos modelos de viscosidade e cinética de cristalização foram obtidos experimentalmente. O método numérico utilizado foi o método dos volumes finitos.

\section{Cinética de Cristalização Quiescente}

A equação que descreve a cinética de cristalização quiescente isotérmica é conhecida como equação de Avrami e possui a seguinte forma ${ }^{[18]}$ :

$$
\theta(\mathrm{t})=1-\exp \left(-\mathrm{kt}^{\mathrm{n}}\right)
$$

onde $\theta(t)$ é a cristalinidade relativa, $\frac{X(t)}{X_{\infty}}$ enquanto $\mathrm{X}(\mathrm{t})$ é o grau de cristalinidade no tempo t e $\mathrm{X}_{\infty}$ é o máximo grau de cristalinidade obtido; $\mathrm{k}$ é uma constante que está relacionada à velocidade de nucleação e crescimento da fase cristalina e n é conhecido como índice de Avrami e expressa o tipo de nucleação e morfologia desenvolvidos na cristalização.

Apesar da teoria de cinética de cristalização isotérmica estar bem desenvolvida, os polímeros, durante o processamento, são submetidos a um resfriamento não isotérmico. Segundo Patel e Spruiell[19], o modelo de cinética de cristalização quiescente nãoisotérmico de Nakamura ${ }^{[11]}$ é um dos modelos mais adequados de cinética de cristalização não-isotérmica para ser utilizado no modelamento matemático de processos de transformação de polímeros. Este modelo consiste numa generalização do modelo de cristalização isotérmica de Avrami, sendo expresso por:

$$
\theta(\mathrm{t})=1-\exp \left[-\left(\int_{0}^{\mathrm{t}} \mathrm{K}(\mathrm{T}) \mathrm{dt}\right)^{\mathrm{n}}\right]
$$

onde $\theta(\mathrm{t})$ é a cristalinidade relativa, $\mathrm{K}(\mathrm{T})$ é um parâmetro de taxa que depende da temperatura, e n é o índice de Avrami. 
A Equação (2) foi desenvolvida assumindo que o número de sítios de nucleação é independente da temperatura, e que todos os sítios são ativados simultaneamente ${ }^{[11]}$.

A dependência de $K(T)$ com a temperatura pode ser obtida pela expressão ${ }^{[15,19]}$ :

$$
K(T)=(\ln 2)^{1 / n}\left(\frac{1}{t_{1 / 2}}\right)
$$

onde n é o índice de Avrami e $\left(\frac{1}{t_{1 / 2}}\right)$ é o inverso do tempo de meia cristalização.

$$
\text { O valor de }\left(\frac{1}{t_{1 / 2}}\right) \text { pode ser obtido pela expressão }
$$

de Hoffman-Lauritzen ${ }^{[14,15]}$ :

$$
\left(\frac{1}{t_{1 / 2}}\right)=\left(\frac{1}{t_{1 / 2}}\right)_{0} \exp \left(-\frac{U^{*} / R}{T-T \infty}\right) \exp \left(-\frac{K_{g}}{T \Delta T f}\right)
$$

onde $\Delta \mathrm{T}=\mathrm{T}_{\mathrm{m}}^{0}-\mathrm{T}, \mathrm{f}=\frac{2 \mathrm{~T}}{\mathrm{~T}+\mathrm{Tm}^{0}}$, que é um fator de correção; $\left(\frac{1}{t_{1 / 2}}\right)_{0}$ é um fator que inclui os termos independentes da temperatura; $\mathrm{Tm}^{0}$ é a temperatura de fusão no equilíbrio, $U^{*}$ é a energia de ativação para o transporte de unidades cristalinas, $\mathrm{R}$ é a constante dos gases, $\mathrm{T}_{\infty}=\mathrm{T}_{\mathrm{g}}-30 \mathrm{~K}$ e $\mathrm{K}_{\mathrm{g}}$ é uma constante dependente do tipo de nucleação.

O modelo de Nakamura não leva em conta o tempo de indução para nucleação. Para determinar o tempo de indução para cristalização quiescente, o método de Sifleet ${ }^{[20]}$ pode ser utilizado. O tempo de indução não-isotérmico pode ser obtido pela soma de tempos de indução isotérmicos de acordo com

$$
\int_{0}^{t_{I}} \frac{d t}{t_{i}(T)}=1
$$

onde $t_{i}$ é o tempo de indução isotérmico e $t_{I} o$ tempo de indução não-isotérmico. Para cristalização a partir do fundido, o tempo de indução isotérmico pode ser obtido a partir da expressão de Godovsky e Slonimsky ${ }^{[21]}$ :

$$
\mathrm{t}_{\mathrm{i}}=\mathrm{t}_{\mathrm{ra}}\left(\mathrm{T}_{\mathrm{m}}^{\mathrm{0}}-\mathrm{T}\right)^{-\mathrm{a}}
$$

onde $\mathrm{t}_{\mathrm{m}}$ e a são constantes independentes da temperatura.

Os parâmetros para se determinar os modelos que descrevem a cinética de cristalização não isotérmica podem ser obtidos experimentalmente através de calorimetria diferencial de varredura (DSC). A metodologia para se determinar os parâmetros do modelo de Nakamura, assim como os tempos de indução, pode ser encontrada nos trabalhos de d'Ávila ${ }^{[2]}$, Chan e Isayev ${ }^{[15]}$ e Patel e Spruiel[ ${ }^{[19]}$.

\section{Modelo de Viscosidade}

No presente trabalho foi utilizado o modelo de viscosidade de Cross Modificado ${ }^{[23]}$. Este modelo é capaz de descrever a dependência da viscosidade com a taxa de cisalhamento tanto para baixos valores, como para altos valores de taxa de cisalhamento. Considerando também a dependência da viscosidade com a temperatura, este modelo pode ser expresso da seguinte forma:

$$
\eta=\frac{\eta_{0}(T)}{1+\left(\frac{\eta_{0}(T) g}{\tau^{*}}\right)^{1-m}}
$$

\section{e}

$$
\eta_{0}(T)=\operatorname{Bexp}\left(\frac{T_{b}}{T}\right)
$$

onde $\eta$ é a viscosidade, $\dot{\gamma}$ é a taxa de cisalhamento, $\eta_{0}$ é a viscosidade newtoniana, $\tau^{*}$ e $m$ são constantes, $B$ e $\mathrm{T}_{\mathrm{b}}$ são constantes e $\mathrm{T}$ é a temperatura.

\section{Formulaçáo Matemática do Processo}

Neste trabalho foi feita a simulação das fases de preenchimento e resfriamento do processo de injeção. Foi considerado que o escoamento é laminar, o fluido é incompressível, as forças gravitacionais são desprezíveis e o escoamento é quase estacionário. A equação constitutiva que relaciona a tensão com a taxa de deformação foi a equação do fluido newtoniano generalizado, expresso por ${ }^{[22]}$ : 


$$
\overline{\bar{\tau}}=-\eta \overline{\bar{\gamma}}+p \overline{\bar{\delta}}
$$

onde $\bar{\tau}$ é o tensor tensão, $\overline{\bar{\gamma}}$ é o tensor taxa de deformação, $\eta$ é a viscosidade dada pelo modelo de Cross Modificado, p é a pressão hidrostática e $\overline{\bar{\delta}}$ é o tensor identidade.

Considerando uma geometria retangular, como mostrada na Figura 1, e as hipóteses simplificativas acima citadas, as equações governantes, para a fase de preenchimento ficam da seguinte forma ${ }^{[10,24]}$ :

- Equação da conservação da massa:

$$
\mathrm{Q}=w \int_{-\frac{\mathrm{h}}{2}}^{\frac{\mathrm{h}}{2}} \mathrm{y}_{\mathrm{x}} \mathrm{dz}
$$

onde $\mathrm{Q}$ é a vazão que é constante e conhecida, $\mathrm{v}_{\mathrm{x}}$ é a velocidade na direção do escoamento, w é a largura do molde e h é a espessura do molde.

- Equação da conservação da quantidade de movimento:

$$
\frac{\partial}{\partial z} \eta\left(\frac{\partial v_{x}}{\partial z}\right)-\frac{\partial p}{\partial \mathrm{x}}=0
$$

onde $\eta$ é a viscosidade que depende da taxa de cisalhamento e da temperatura, $\mathrm{v}_{\mathrm{x}}$ é a velocidade na direção do escoamento e p é a pressão.

- Equação da conservação da energia:

$$
\rho \mathrm{C}_{\mathrm{p}}\left(\frac{\partial \mathrm{T}}{\partial \mathrm{t}}+\mathrm{v}_{\mathrm{x}} \frac{\partial \mathrm{T}}{\partial \mathrm{x}}\right)=\mathrm{k} \frac{\partial^{2} \mathrm{~T}}{\partial \mathrm{z}^{2}}+\eta \dot{\gamma}^{2}+\dot{\mathrm{H}}
$$

onde $\rho$ é a densidade, T é a temperatura, $\eta$ é a viscosidade e $\dot{\gamma}$ é a taxa de cisalhamento; $\mathrm{C}_{\mathrm{p}}$ é o calor específico a pressão constante e ké a condutividade térmica, que foram considerados como sendo constantes.

O termo Ḣ na equação (12) incorpora o calor de cristalização e pode ser descrito como ${ }^{[13,15]}$ :

$$
\dot{\mathrm{H}}=\Delta \mathrm{H}_{\mathrm{f}} \mathrm{X} \infty \frac{\partial \theta}{\partial t}
$$

onde $\Delta \mathrm{H}_{\mathrm{f}}$ é calor latente de fusão de um cristal perfeito, $X_{\infty}$ é máximo grau de cristalinidade do

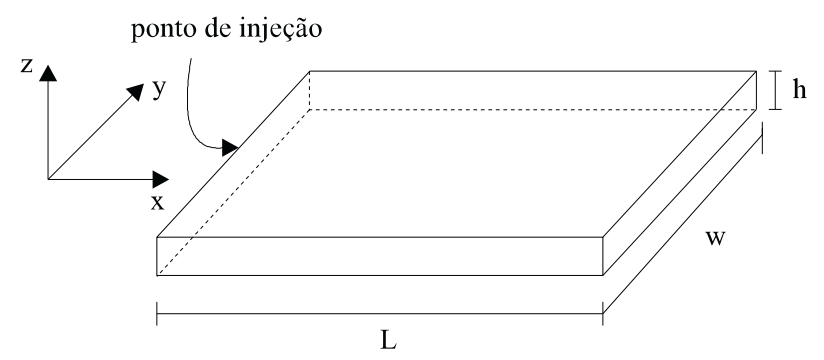

Figura 1: Geometria considerada no presente trabalho.

polímero e $\frac{\partial \theta}{\partial t}$ é a taxa de cristalização que pode ser obtida pelo modelo de Nakamura (Equação 2).

A equação (2) pode ser colocada na forma diferencial, a fim de ser utilizada na simulação, ou seja:

$$
\frac{\mathrm{d} \theta}{\mathrm{dt}}=\mathrm{nK}(\mathrm{T})(1-\theta)[-\ln (1-\theta)]^{\frac{\mathrm{n}-1}{\mathrm{n}}}
$$

Para a fase de preenchimento as seguintes condições iniciais e de contorno foram consideradas ${ }^{[2]}$ :

1) $\mathrm{Na}$ entrada ou ponto de injeção $(x=0)$ a temperatura é uniforme e igual a temperatura do fundido e o escoamento pode ser considerado como plenamente desenvolvido, portanto,

$$
\begin{aligned}
& \mathrm{v}_{\mathrm{x}}(0, \mathrm{z}, \mathrm{t})=\mathrm{v}_{0}(\mathrm{z}) \\
& \mathrm{T}(0, \mathrm{z}, \mathrm{t})=\mathrm{T}_{0}
\end{aligned}
$$

2) Nas paredes do molde a velocidade é nula e a temperatura do molde é prescrita, portanto,

$$
\begin{aligned}
& \mathrm{v}_{\mathrm{x}}(\mathrm{x}, \mathrm{h} / 2, \mathrm{t})=0 \\
& \mathrm{~T}(\mathrm{x}, \mathrm{h} / 2, \mathrm{t})=\mathrm{T}_{\mathrm{w}}
\end{aligned}
$$

Assumindo simetria em $\mathrm{z}=0$, tem-se,

$$
\frac{\partial v_{x}}{\partial z}(\mathrm{x}, 0, \mathrm{t})=0
$$

$$
\frac{\partial T}{\partial z}(\mathrm{x}, 0, \mathrm{t})=0
$$

3) A frente de fluxo é plana e avança uniformemente, sendo a temperatura constante e igual à temperatura da linha de centro da região imediatamente anterior à frente de fluxo. Esta condição permite fazer uma aproximação ao fenômeno do fluxo em fonte (fountain flow) na determinação do campo de temperaturas pelo fato de estar sendo considerado que os elementos de fluido na região central da cavidade, 
próximo à frente de fluxo, estão transportando calor em direção às paredes do molde ${ }^{[2,24]}$.

Para a fase de resfriamento, pelo fato de não haver mais o fluxo do material, a equação governante pode ser expressa por:

$$
\rho \mathrm{C}_{\mathrm{P}} \frac{\partial \mathrm{T}}{\partial \mathrm{t}}=\mathrm{k} \frac{\partial^{2} \mathrm{~T}}{\partial \mathrm{z}^{2}}+\dot{\mathrm{H}}
$$

Como condição de contorno foi considerado que a temperatura do molde é prescrita e que existe simetria na linha de centro (Equações 18 e 20)

As equações de conservação simplificadas foram manipuladas para ficarem numa forma adequada para realizar a solução numérica de maneira mais simples. Essas manipulações foram feitas seguindo o procedimento feito por Hieber ${ }^{[24]}$, chegando às seguintes relações:

$$
\begin{aligned}
& \dot{\gamma}=\frac{\Delta \mathrm{z}}{\eta} \\
& \Lambda=\frac{\mathrm{Q}}{2 \mathrm{wS}} \\
& \mathrm{S}_{\mathrm{f}} \equiv \int_{0}^{\frac{\mathrm{h}}{2}} \frac{\mathrm{z}^{2}}{\eta} \mathrm{dz}
\end{aligned}
$$

onde $\dot{\gamma}$ é a taxa de cisalhamento, $\eta$ é a viscosidade, $\Lambda \equiv-\frac{\partial \mathrm{p}}{\partial \mathrm{z}}$ e $\mathrm{S}_{\mathrm{f}}$ é conhecido por fluidez do fundido.

\section{Procedimento Computacional}

O método numérico utilizado neste trabalho foi o método dos volumes finitos ${ }^{[25,26]}$. A escolha deste método foi feita pelo fato deste ser bastante adequado para resolver problemas relacionados com transferência de calor e mecânica dos fluidos ${ }^{[2]}$.

\section{Discretização da Equação da Energia}

Para realizar a simulação numérica utilizando o método dos volumes finitos é necessário discretizar a equação diferencial que se deseja obter a solução. No caso deste trabalho, foi necessário discretizar a

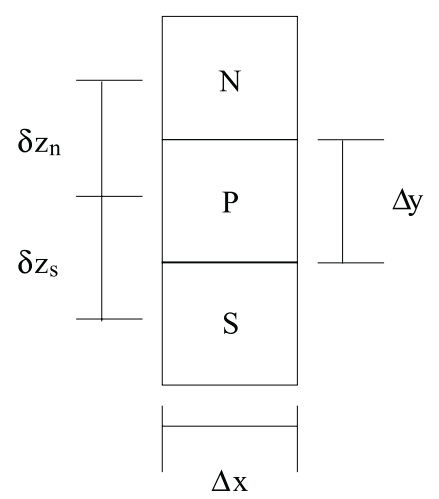

Figura 2. Volume de controle da equação discretizada.

equação da energia (Equação 12).

$\mathrm{O}$ procedimento adotado foi o de considerar o termo de convecção como termo fonte ${ }^{[2,27]}$, assim como os termos de dissipação viscosa e cristalização ${ }^{[2]}$. Portanto, a solução numérica do problema foi obtida resolvendo um problema de condução unidimensional transiente com termo fonte. Sendo o termo fonte, $S=S_{\text {con }}+S_{\text {vise }}+S_{\text {crist }}$ onde $S_{\text {conv }}, S_{\text {visc }}$ e $\mathrm{S}_{\text {crist }}$ são os termos de convecção, dissipação viscosa e cristalização, respectivamente, este pode ser linearizado da seguinte forma ${ }^{[25,26]}$ :

$$
\mathrm{S}=\mathrm{S}_{\mathrm{C}}+\mathrm{S}_{\mathrm{P}} \mathrm{T}_{\mathrm{P}}
$$

onde $\mathrm{S}_{\mathrm{c}}$ e $\mathrm{S}_{\mathrm{p}}$ são respectivamente, coeficientes linear e angular de uma reta e $T_{p}$ é o valor da temperatura no volume $\mathrm{P}$ (ver Figura 2).

Uma descrição detalhada dos procedimentos de discretização (integração da equação) do problema de condução unidimensional transiente, assim como a linearização do termo fonte pode ser encontrada em Patankar ${ }^{[25]}$ e Maliska $^{[26]}$. O volume de controle para a equação discretizada pode ser visto na Figura 2.

A equação da conservação da energia discretizada em volumes finitos, para uma formulação implícita no tempo ${ }^{[25,26]}$, apresenta a seguinte forma:

$$
\mathrm{A}_{\mathrm{P}} \mathrm{T}_{\mathrm{P}}=\mathrm{A}_{\mathrm{N}} \mathrm{T}_{\mathrm{N}}+\mathrm{A}_{\mathrm{S}} \mathrm{T}_{\mathrm{S}}+\mathrm{B}
$$

$$
\begin{aligned}
& \text { onde } A_{\mathrm{F}}=\frac{\mathrm{k}}{\mathrm{C}_{\mathrm{F}}(\delta \mathrm{z})_{\mathrm{h}}}, A_{g}=\frac{\mathrm{k}}{\mathrm{C}_{\mathrm{F}}(\delta \mathrm{z})_{\mathrm{S}}} \text {, } \\
& \mathrm{B}=\mathrm{S}_{\mathrm{C}} \Delta z+A_{\mathrm{F}}^{0} \mathrm{~T}_{\mathrm{F}}^{\circ}, A_{\mathrm{F}}^{\circ}=\frac{\rho \Delta z}{\Delta t} \mathrm{e} \\
& A_{\mathrm{F}}=A_{\mathrm{N}}+A_{B}+A_{\mathrm{F}}^{\circ}-\mathrm{S}_{\mathrm{F}} \Delta
\end{aligned}
$$

Os subescritos $\mathrm{P}, \mathrm{N}$ e $\mathrm{S}$ indicam que o valor do coeficiente é tomado nos volumes $\mathrm{P}, \mathrm{N}$ e $\mathrm{S}$, respectivamente (ver Figura 2). O sobrescrito 0 indica o valor tomado no passo de tempo anterior. 
O termo fonte $\mathrm{S}$, de acordo com a equação (25) possui a seguinte forma ${ }^{[2]}$ :

$$
S=\left[\left(\frac{\eta \dot{Y}^{2}}{C_{F}}\right)+\left(\rho \frac{v_{n} T P^{*}}{\Delta \mathrm{r}}\right)+\left(\Delta H_{f} X_{w} \frac{\partial \theta}{\partial t}\right)\right]-\left[\rho\left(\frac{v_{n}}{\Delta x}\right)\right] T_{F}
$$

onde $\mathrm{TP}^{*}$ é o valor da temperatura do volume vizinho localizado à montante do volume $\mathrm{P}$ na direção do escoamento.

As condições de contorno foram aplicadas nos volumes de fronteira utilizando a técnica de meio volume de controle na fronteira, como mostrado em Patankar ${ }^{[25]}$. Assim, os valores dos coeficientes da equação (26) para a fronteira norte (parede do molde) fo$\operatorname{ram} A_{N}=A_{S}=0, A_{P}=1$ e $B=T_{W}$. Para a fronteira sul (linha de centro), os valores dos coeficientes obtidos foram $A_{N}=A_{P}=1$ e $A_{S}=B=0$.

Assim, para cada volume obteve-se os coeficientes $\mathrm{A}_{\mathrm{i}}$ e $\mathrm{B}$. Esses termos consistem nos coeficientes de um sistema linear, onde a solução desse sistema fornece os valores de $T_{p}$ para cada volume, obtendo assim a solução aproximada para o problema.

$\mathrm{Na}$ fase de resfriamento, a única diferença para a solução por volumes finitos está no termo fonte, onde só existe o termo de cristalização $\mathrm{S}_{\text {crist }}{ }^{[2]}$.

\section{Procedimentos de Cálculo}

O programa computacional foi desenvolvido em linguagem C utilizando microcomputador PC-486, onde a solução do sistema linear, obtido pela discretização por volumes finitos, foi feita utilizando o método iterativo de Jacobi ${ }^{[26]}$. Foi utilizada uma malha igualmente espaçada nas direções do fluxo e na direção da espessura. A posição da frente de fluxo foi determinada fazendo-se uma relação entre o passo de tempo e $\Delta$ t a posição da frente, ou seja,

$$
\Delta t=\frac{\mathrm{L}}{\mathrm{N}_{1} \mathrm{U}}
$$

e

$$
\Delta x=\delta x=\frac{L}{N_{1}}
$$

onde $\mathrm{U}=\mathrm{Q} / \mathrm{hw}$ e $\mathrm{N}_{1}$ é o número de passos de tempo.

O procedimento para resolver o problema das fases de preenchimento e resfriamento do processo de injeção foi o descrito a seguir ${ }^{[2]}$ :
1) No primeiro passo de tempo a temperatura foi assumida como uniforme (Equação 16), e o perfil de velocidades foi assumido como plenamente desenvolvido (Equação 15) onde foi utilizada a solução analítica para um fluido de lei das potências ${ }^{[10]}$.

2) Avança-se um passo de tempo $\Delta$ t e calcula-se o campo de temperaturas para todos os volumes.

3) Conhecido o novo campo de temperaturas calcula-se, utilizando os valores obtidos de $\dot{y}$ do passo de tempo anterior, os valores de $\eta$ (Equação 7 e 8), $S_{f}$ (Equação 24) e $\Lambda$ (Equação 23).

4) Calcula-se os valores de $\dot{\gamma}$ (Equação 22) e, por integração numérica de $\Lambda$ e , $\dot{\gamma}$ determina-se os campos de pressão e velocidade.

5) Com os novos valores de $\eta$, $\dot{y}$ e $v_{x}$, calcula-se novamente o campo de temperaturas e retorna-se ao item 3 até atingir a convergência com uma tolerância estabelecida.

6) A frente de fluxo avança um volume na direção do fluxo, considerando como possuindo temperatura uniforme e igual à temperatura da linha de centro do campo de temperaturas calculado.

7) Retorna-se ao ítem 2 até o preenchimento do molde.

8) Calcula-se o campo de temperaturas para a fase de resfriamento até um tempo de resfriamento determinado.

\section{Procedimento Experimental}

\section{Material}

O material utilizado foi um polipropileno isotático fabricado pela OPP em planta piloto. Segundo o fornecedor, este material possui peso molecular igual a $470000 \mathrm{~g} / \mathrm{mol}$.

\section{Reometria}

Foram feitos ensaios de reometria para obter a relação da viscosidade com a taxa de cisalhamento e temperatura. Para baixas taxas de cisalhamento $(0,02$ - 1,0 $\mathrm{s}^{-1}$ ) foi utilizado um reômetro de placas paralelas ARES da Rheometric Scientific. Para taxas de cisalhamento altas $\left(10-5000 \mathrm{~s}^{-1}\right)$ foi utilizado um reômetro capilar Instron 3211.

Os ensaios foram realizados em três temperaturas diferentes: $200^{\circ} \mathrm{C}, 215^{\circ} \mathrm{C}$ e $230^{\circ} \mathrm{C}$ a fim de se conseguir obter a dependência da viscosidade com a temperatura.

Com os dados experimentais foram obtidas as curvas do modelo de Cross modificado. 


\section{Calorimetria Diferencial de Varredura (DSC)}

Os experimentos de DSC foram realizados para determinar os parâmetros do modelo de cinética de cristalização de Nakamura e os tempos de indução. O equipamento utilizado foi o DSC-7 da Perkin Elmer.

Foram feitas amostras de aproximadamente $10 \mathrm{mg}$ que foram seladas em panelas de alumínio e as corridas foram realizadas em atmosfera de nitrogênio.

Cada amostra foi aquecida até $200^{\circ} \mathrm{C}$ e mantida nesta temperatura por 5 minutos para eliminar a história térmica do material. Posteriormente, cada amostra foi levada, com uma taxa de resfriamento de $200{ }^{\circ} \mathrm{C} / \mathrm{min}$, para a temperatura de cristalização, onde foi realizada a cristalização isotérmica. As temperaturas de cristalização utilizadas foram de: $118^{\circ} \mathrm{C}$, $122{ }^{\circ} \mathrm{C}, 126^{\circ} \mathrm{C}$ e $130{ }^{\circ} \mathrm{C}$.

As amostras foram novamente aquecidas para a temperatura de $200{ }^{\circ} \mathrm{C}$ por 5 minutos e posteriormente resfriadas em diferentes taxas de resfriamento. As taxas utilizadas foram: $5^{\circ} \mathrm{C} / \mathrm{min}, 10^{\circ} \mathrm{C} / \mathrm{min}, 20^{\circ} \mathrm{C} / \mathrm{min}$ e $40^{\circ} \mathrm{C} / \mathrm{min}$.

A partir dos resultados fornecidos pelo DSC para a cristalização isotérmica foi possível determinar os parâmetros da equação de Nakamura e determinar o tempo de indução. Os resultados de cristalização não isotérmica foram utilizados para a comparação entre os resultados do modelo de Nakamura com os resultados experimentais.

\section{Resultados e Discussões}

\section{Reometria}

Os parâmetros obtidos para o modelo de Cross Modificado (Equação 7 e 8 ) foram $m=0,31, \tau^{*}=2,92$ x $10^{4} \mathrm{~Pa}, \mathrm{~B}=0,22 \mathrm{~Pa} . \mathrm{s}$ e $\mathrm{T}_{\mathrm{b}}=5,16 \times 10^{3} \mathrm{~K}$. Os valores de viscosidade em função da taxa de cisalhamento obtidos pelo modelo de Cross modificado pode ser visto na Figura 3. Verifica-se que este modelo consegue ajustar de maneira satisfatória os valores experimentais, para baixas taxas de cisalhamento, além dos valores para altas taxas de cisalhamento.

\section{Cinética de Cristalização}

A análise de cinética de cristalização isotérmica de Avrami (Equação 1) foi feita para determinar os tempos de meia cristalização $t_{1 / 2}$ e o índice de Avrami n. A Tabela 1 mostra os valores obtidos através da análise de área parcial dos picos de cristalização em corridas isotérmicas no DSC para diferentes temperaturas de cristalização.

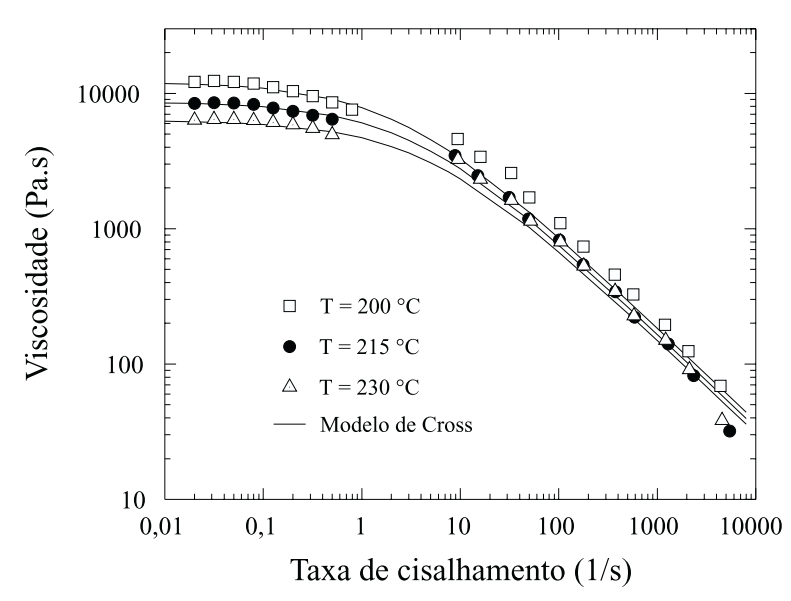

Figura 3. Gráfico da viscosidade em função da taxa de cisalhamento em diferentes temperaturas. Símbolos representam os valores experimentais, linhas representam os valores obtidos pelo modelo de Cross modificado.

Pode-se observar pelo coeficiente de correlação r, que foi obtido um bom ajuste do modelo de Avrami com os pontos experimentais. $\mathrm{O}$ valor do índice de Avrami variou entre 2,1 e 2,3. Portanto, para o modelo de Nakamura foi utilizado um valor de $n$ igual a 2,2.

Com os dados de cinética de cristalização isotérmica foi possível determinar $\mathrm{K}(\mathrm{T})$ da equação de Nakamura (Equação 2) através da determinação dos tempos de meia cristalização pela equação de Hoffman-Lauritzen (Equação 3 e 4). A Figura 4 mostra um gráfico com os pontos experimentais e a reta obtida por regressão linear, onde foram obtidos os parâmetros da equação de HoffmanLauritzen. Os valores fundamentais para o polipropileno ${ }^{[12]}$ utilizados foram $\mathrm{U}^{*}=6284 \mathrm{~J} / \mathrm{mol}, \mathrm{R}=$ $8,3143 \mathrm{~J} /(\mathrm{molK}), \mathrm{T} \infty=230 \mathrm{~K} \mathrm{e} \mathrm{Tm}{ }^{0}=445 \mathrm{~K}$.

Os valores obtidos de $\left(\frac{1}{t_{1 / 2}}\right)_{0}$ e $K_{g}$ obtidos por regressão foram $1,67 \times 10^{7} \mathrm{~s}^{-1}$ e $3,16 \times 10^{5} \mathrm{~K}^{2}$, respectivamente. O coeficiente de correlação da reta foi - 0,9775 , mostrando um ajuste razoável da reta com os valores experimentais, como pode ser observado na Figura 4.

O tempo de indução para início da cristalização nãoisotérmico foi obtido pela equação de Sifleet (Equação 5), que relaciona o tempo de indução não-isotérmico com o tempo de indução isotérmico, que pode ser descrito pela equação de Godovsky-Slonimsky (Equação 6).

Tabela 1. Valores obtidos dos parâmetros da equação de Avrami.
\begin{tabular}{ccccc}
\hline $\mathbf{T}\left({ }^{\circ} \mathbf{C}\right)$ & $\mathbf{k}\left(\mathbf{1} / \mathbf{s}^{\mathbf{n}}\right)$ & $\mathbf{n}$ & $\mathbf{t}_{1 / 2}(\mathbf{s})$ & $\mathbf{r}$ \\
\hline 118 & 1,54 & 2,1 & 40,8 & 0,99997 \\
122 & $5,44 \times 10-2$ & 2,1 & 204,4 & 0,9993 \\
126 & $4,72 \times 10-3$ & 2,3 & 512,3 & 0,99996 \\
130 & $7,03 \times 10-4$ & 2,3 & $1,27 \times 103$ & 0,9998 \\
\hline
\end{tabular}




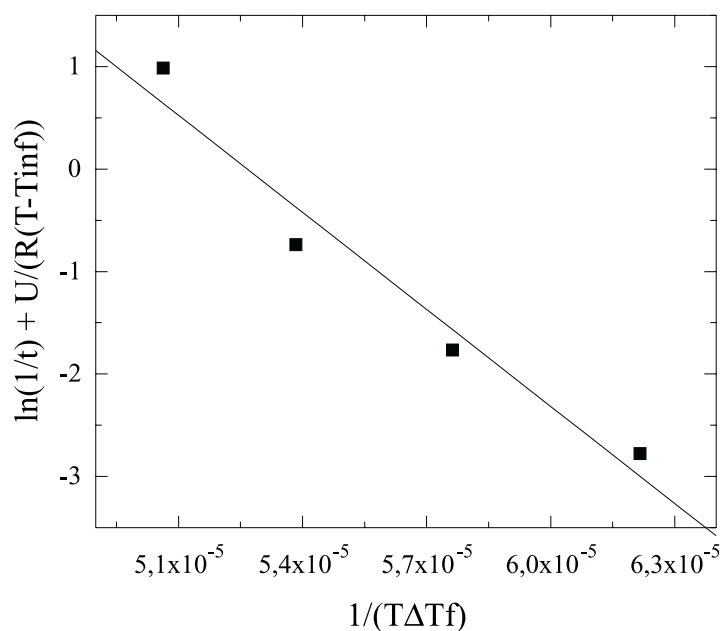

Figura 4. Gráfico para determinação dos parâmetros da equação de Hoffmann-Lauritzen. Símbolos são os valores experimentais e a linha representa a reta obtida por regressão linear dos pontos.

Os valores experimentais dos tempos de indução isotérmico em diferentes temperaturas foram obtidos através dos dados de DSC, onde o tempo de indução é considerado como o tempo necessário para o início da cristalização. A partir dos dados experimentais foram obtidos os parâmetros da equação de Godovsky-Slonimsky. Os valores de $t_{m}$ e a obtidos foram $4,52 \times 10^{8} \mathrm{SK}^{4,1} \mathrm{e} 4,1$, respectivamente. A partir desses resultados foi possível comparar os tempos de indução, obtidos através dos ensaios com taxa de resfriamento no DSC, com os valores obtidos pela equação de Sifleet (ver Figura 5).

Conhecendo-se os tempos de indução não isotérmico e os parâmetros da equação de Nakamura foi possível descrever a cinética de cristalização nãoisotérmica do material estudado. A comparação dos resultados experimentais em diferentes taxas de

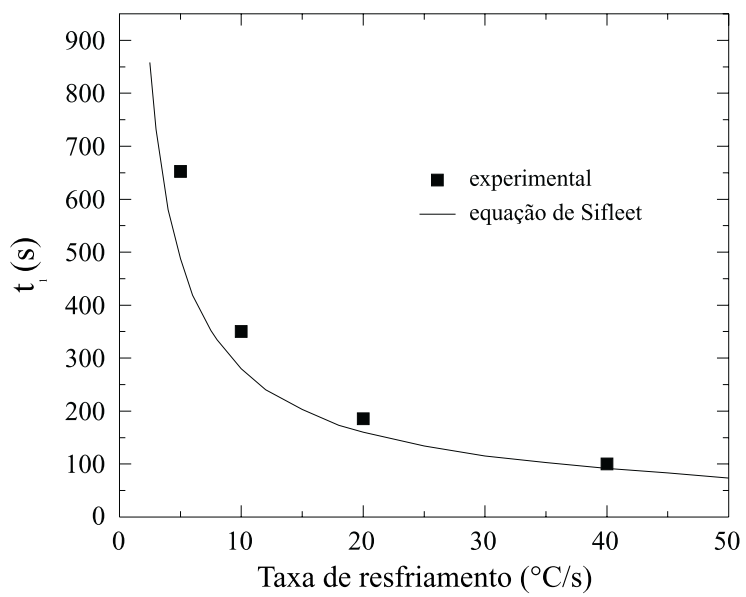

Figura 5. Gráfico de tempo de indução não-isotérmico versus taxa de resfriamento. Símbolos representam valores experimentais, linha representa a equação de Sifleet.

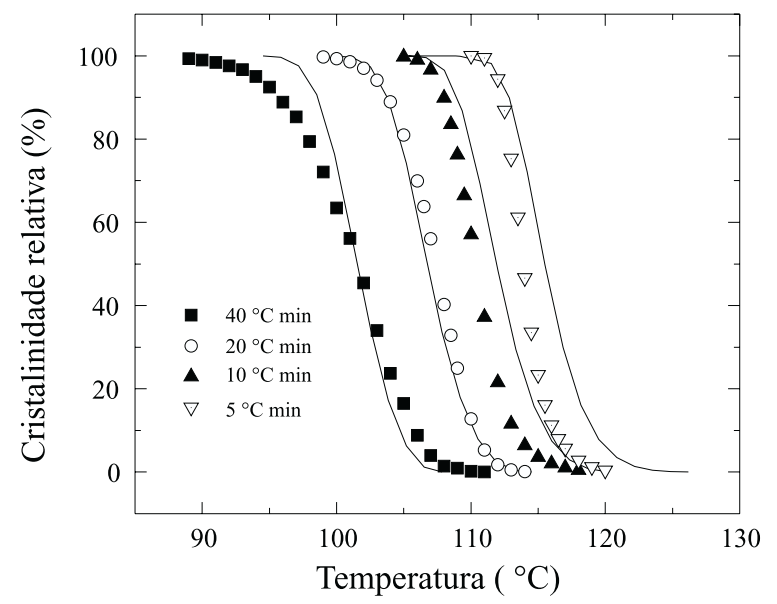

Figura 6. Gráfico de cristalinidade relativa versus temperatura em diferentes taxas de resfriamento. Símbolos representam os valores experimentais, linhas representam os valores obtidos pelo modelo de Nakamura considerando os tempos de indução.

resfriamento com os valores obtidos pelo modelo de Nakamura está mostrado na Figura 6.

Pode-se observar pela Figura 6 que foi obtida uma concordância razoável entre os valores teóricos com os experimentais, principalmente para a taxa de resfriamento igual a $20^{\circ} \mathrm{C} / \mathrm{min}$. As maiores discrepâncias entre os valores experimentais com os obtidos pelo modelo de Nakamura foram observadas em taxas de resfriamento menores $\left(5^{\circ} \mathrm{C} / \mathrm{min}\right.$ e $\left.10^{\circ} \mathrm{C} / \mathrm{min}\right)$.

Deve-se ressaltar, no entanto, que no processo de injeção, as taxas de resfriamento são muito maiores do que as taxas utilizadas para determinar os parâmetros do modelo. Porém, as baixas taxas de resfriamento do DSC constituem uma grande limitação para descrever a cinética de cristalização não-isotérmica em condições de processamento, sendo necessário fazer grandes extrapolações na simulação do processo de injeção.

\section{Fase de Preenchimento}

Para a simulação da fase de preenchimento foram utilizados os parâmetros do material (modelo de viscosidade de Cross modificado e cinética de cristalização não-isotérmica) obtidos experimentalmente, cujos valores estão nas seções anteriores. Os valores de $\rho, C_{p}$ , $\mathrm{k}, \Delta \mathrm{H}_{\mathrm{f}}$ e $\mathrm{X}_{\infty}$ para o polipropileno utilizados foram $900 \mathrm{~kg} / \mathrm{m}^{3}, 2140 \mathrm{~J} /(\mathrm{kg} . \mathrm{K}), 0,193 \mathrm{~J} /($ s.m.K), 2,09x10 J/ $\mathrm{kg}$ e 0,4 , respectivamente ${ }^{[2]}$. A temperatura do fundido $\mathrm{T}_{0}$ e a temperatura do molde $\mathrm{T}_{\mathrm{w}}$ considerados foram $230^{\circ} \mathrm{C}$ e $25^{\circ} \mathrm{C}$, respectivamente. Os valores de $\mathrm{L}, \mathrm{w}$ e $\mathrm{h} /$ 2 foram de $15 \mathrm{~cm}, 2,5 \mathrm{~cm}$ e $1,5 \mathrm{~mm}$, respectivamente.

A Figura 7 mostra a distribuição de temperatura, representada por linhas isotérmicas em graus centígrados, para uma vazão igual a $50 \mathrm{~cm}^{3} / \mathrm{s}$, no momento em 
que o molde acabou de ser preenchido. No eixo do comprimento (eixo horizontal), $\mathrm{x}=0$ indica o ponto de injeção, e no eixo da espessura (eixo vertical), $\mathrm{z}=0$ indica o centro da cavidade. Pode-se observar, pela Figura, os grandes gradientes de temperatura na região próxima à parede do molde. Isto ocorre devido à grande diferença entre as temperaturas do molde e do fundido. Sendo a temperatura de solidificação do polipropileno igual a $160^{\circ} \mathrm{C}^{[9]}$, pode-se verificar que ocorre a formação de uma camada solidificada durante o preenchimento do molde. Na região central verifica-se uma região de temperatura igual à temperatura de entrada devido à baixa condutividade térmica do polímero. Ainda é possível observar por esta Figura o efeito do fluxo em fonte considerado a partir da condição de temperatura uniforme na frente de fluxo. Este efeito pode ser observado pelo fato de, ao longo do eixo $\mathrm{x}$ da Figura, as linhas isotérmicas passarem por um mínimo na direção da espessura em uma posição $\mathrm{x}$ mais próxima do ponto de injeção. Se esta condição não fosse utilizada, as linhas isotérmicas não passariam por esse mínimo pois não estaria sendo considerado o efeito de que os elementos de fluido do centro da cavidade próximo à frente de fluxo estariam transportando calor do centro da cavidade para as paredes do molde.

A Figura 8 mostra um gráfico de temperatura versus espessura, para diferentes vazões, na posição do comprimento $x=7,5 \mathrm{~cm}$. Observa-se que, para uma mesma espessura, a temperatura diminui com o aumento do tempo de preenchimento devido ao maior tempo para a troca de calor por condução. Na curva

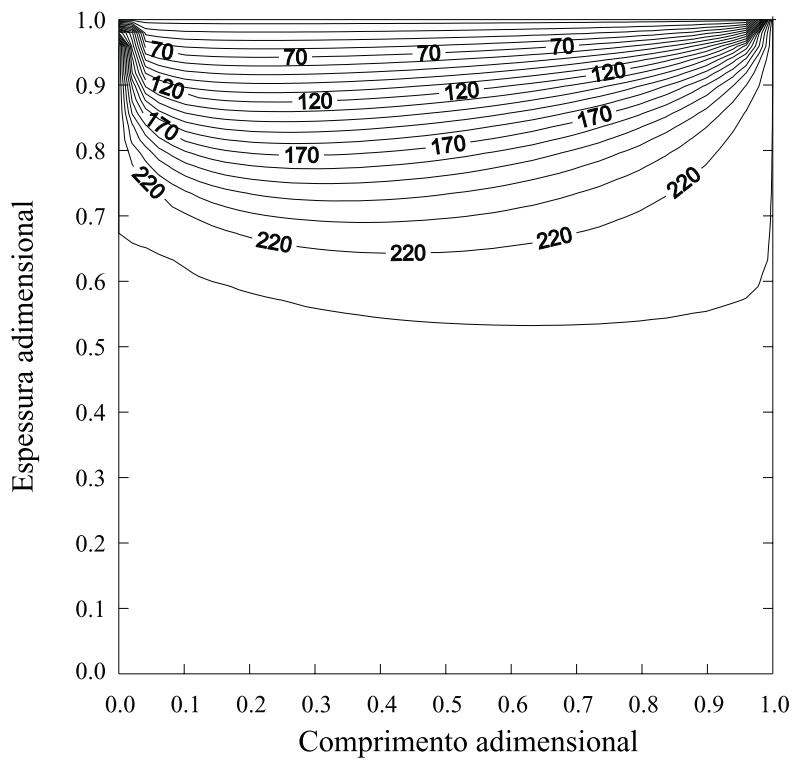

Figura 7. Isotermas $\mathrm{em}{ }^{\circ} \mathrm{C}$ para $\mathrm{Q}=50 \mathrm{~cm}^{3} / \mathrm{s}$ no momento em que o molde acabou de ser preenchido.

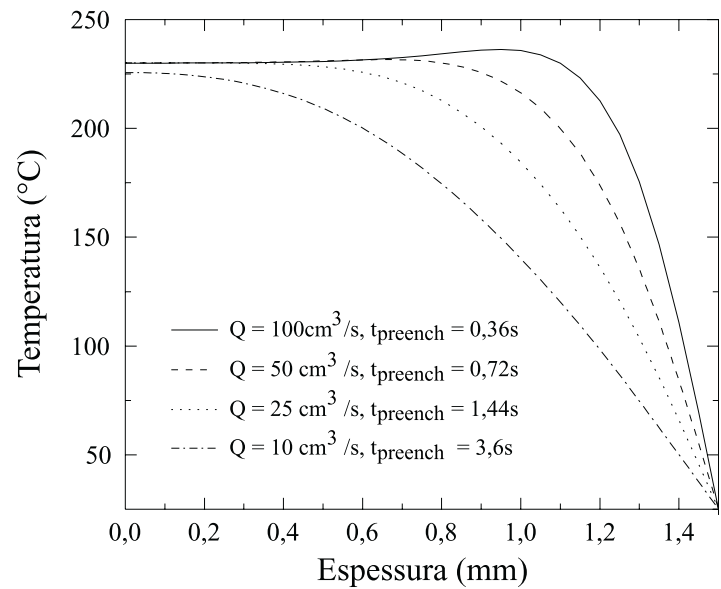

Figura 8. Gráfico de temperatura versus espessura para diferentes vazões na posição do comprimento $\mathrm{x}=7,5 \mathrm{~cm}$.

de $\mathrm{Q}=100 \mathrm{~cm}^{3} / \mathrm{s}$, devido aos menores tempos de resfriamento e aos maiores valores de taxa de cisalhamento (ver Figura 9), pode-se observar o efeito da dissipação viscosa na Figura 8 pelo fato dos valores de temperatura, na região onde a taxa de cisalhamento assume os maiores valores, serem maiores que a temperatura de injeção $T_{0}$.

A Figura 10 mostra curvas de velocidade em função da espessura para diferentes vazões na posição do comprimento $\mathrm{x}=7,5 \mathrm{~cm}$. Pode-se observar que, devido ao aumento da vazão, aumentam os valores de velocidade devido aos menores tempos de preenchimento. Todas as curvas desta Figura apresentam uma região de velocidade igual a zero próxima à parede do molde. Este efeito mostra que o material não está mais escoando nesta região, ou seja, solidificou-se ${ }^{[9]}$.

A variação da pressão em função da posição na direção do escoamento, como apresentada na Figura 11 , mostra que os valores de pressão são maiores para

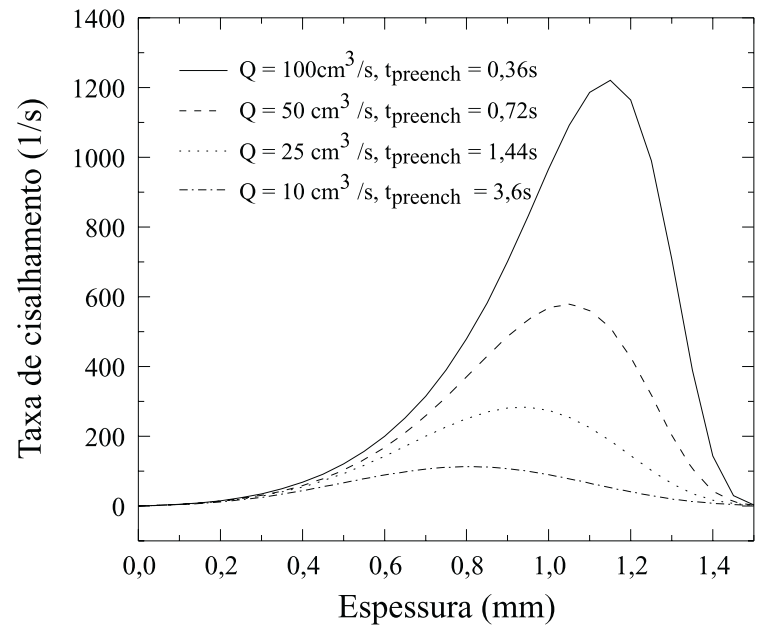

Figura 9. Gráfico de taxa de cisalhamento versus espessura para diferentes vazões na posição do comprimento $\mathrm{x}=7,5 \mathrm{~cm}$. 


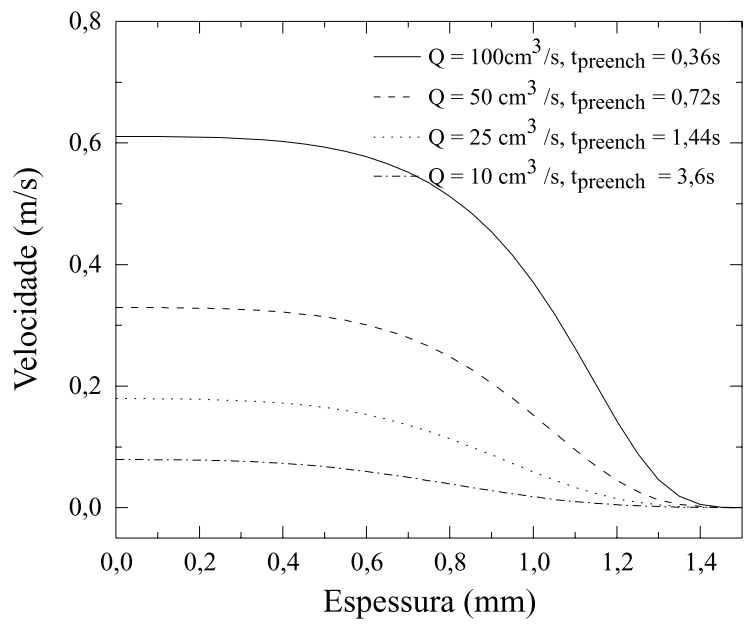

Figura 10. Gráfico de velocidade versus espessura para diferentes vazões na posição do comprimento $\mathrm{x}=7,5 \mathrm{~cm}$.

vazões maiores, como constatado por Kamal e Lafleur ${ }^{[7]}$ para o caso do polietileno de alta densidade. $\mathrm{O}$ fato das curvas da Figura 11 não serem lineares mostra o caráter não-isotérmico do escoamento ${ }^{[7,8]}$.

\section{Fase de Resfriamento}

Foi estudada a fase de resfriamento para as mesmas condições de temperatura do molde e do fundido. A vazão utilizada para a fase de preenchimento foi de $50 \mathrm{~cm}^{3} /$ s. A malha utilizada foi de 31 volumes na direção do escoamento e 31 volumes na direção da espessura.

A Figura 12 mostra um gráfico de temperatura versus espessura para diferentes tempos de resfriamento na posição $\mathrm{x}=7,5 \mathrm{~cm}$, onde pode-se observar o resfriamento do material dentro da cavidade. Com a diminuição da temperatura, devido a troca de calor por condução, a cristalização pode iniciar-se. Observa-se, pela Figura 13, o aumento do grau de

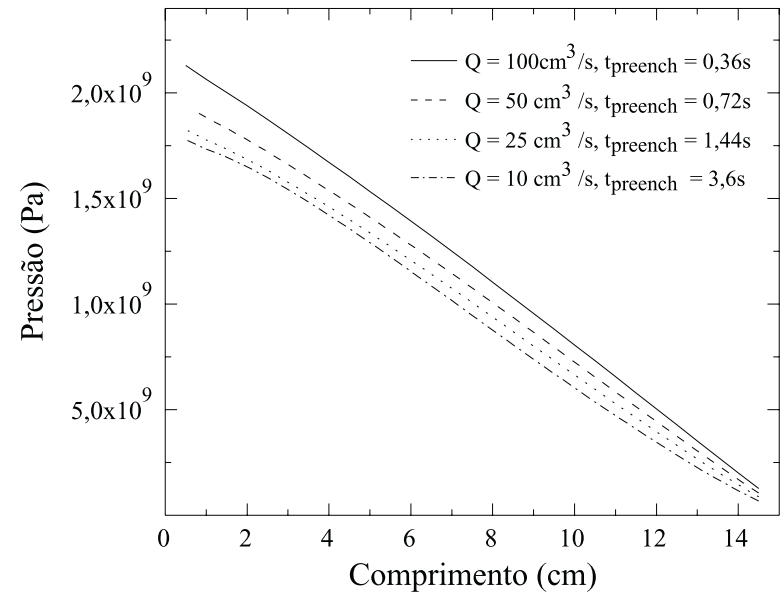

Figura 11. Gráfico da pressão versus posição na direção do escoamento para diferentes vazões. cristalinidade ao longo da espessura com o resfriamento do material. Para o tempo de 0,72 s, que corresponde ao final da fase de preenchimento, pode-se observar que apenas uma pequena região próxima à parede do molde iniciou a cristalização, pois apenas nessa região foram atingidas temperaturas para o início da cristalização. Como o polipropileno é um polímero que cristaliza muito rapidamente ${ }^{[12]}$, verifica-se um grande gradiente de cristalinidade nas regiões entre cristalinidade relativa igual a 0,0 e 1,0 . Pode-se observar também, que $15,72 \mathrm{~s}$ de resfriamento foi o suficiente para o polipropileno cristalizar completamente.

\section{Conclusões}

Dentro do que foi apresentado neste trabalho foi possível chegar às seguintes conclusões:

1) A simulação do processo de injeção do polipropileno permitiu obter resultados dos campos de temperatura, pressão, velocidade, taxa de cisalhamento e cristalinidade em diferentes condições de processamento, onde método dos volumes finitos mostrou-se adequado para determinar a solução numérica da equação da energia na simulação das fases de preenchimento e resfriamento.

2) A caracterização do material foi feita utilizando técnicas conhecidas de caracterização de polímeros (DSC e reometria), mostrando uma relativa facilidade de se obter os parâmetros dos modelos utilizados.

3) Como os parâmetros do modelo de Nakamura são obtidos em taxas de resfriamento bem inferiores ao observado no processo de injeção, existe a necessidade de uma grande extrapolação do modelo para este

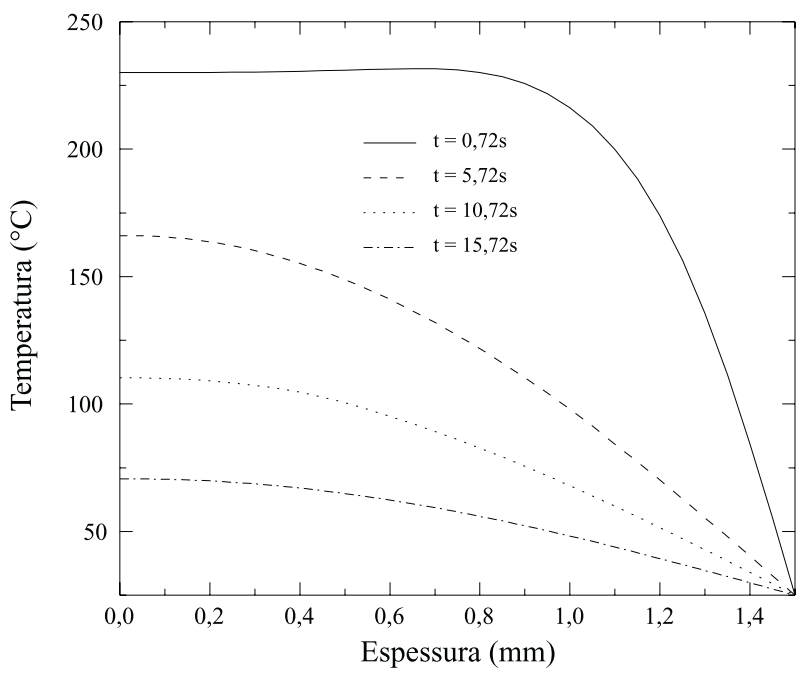

Figura 12. Gráfico de temperatura versus espessura para diferentes tempos de resfriamento. 


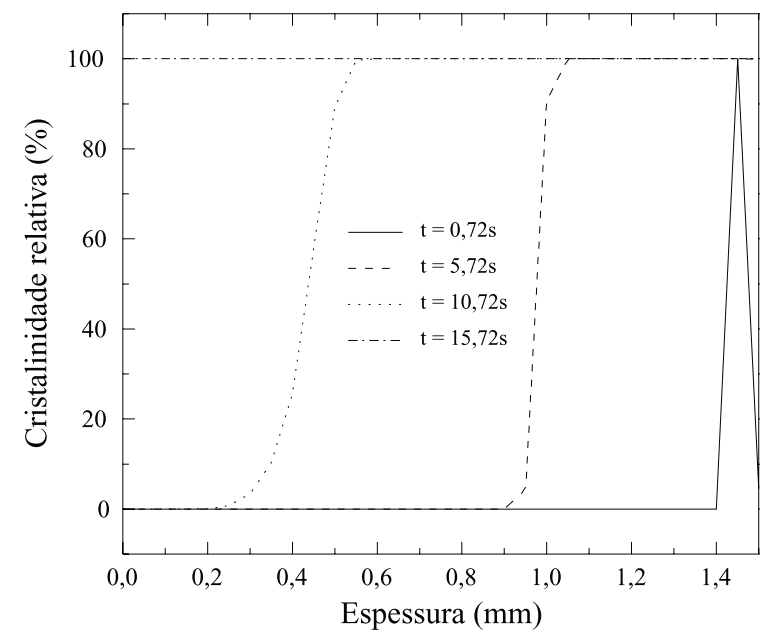

Figura 13. Gráfico de cristalinidade relativa versus espessura para diferentes tempos de resfriamento.

ser utilizado na simulação. Assim, mesmo para a região onde o polímero cristaliza-se de forma quiescente, existe a possibilidade da cinética de cristalização descrita pelo modelo de Nakamura não estar concordando com a cinética de cristalização real do polímero.

\section{Agradecimentos}

Os autores agradecem à FAPESP (94/9317-9) e à CAPES, pelo apoio financeiro.

\section{Referências Bibliográficas}

1. Tadmor, Z.; Gogos, C.; "Principles of Polymer Processing"; John Wiley \& Sons - New York; (1979)

2. d'Ávila, M. A.; "Processo de Moldagem por Injeção de Polímeros Semicristalinos - Caracterização e Simulação Assistida por Computador"; Dissertação de mestrado; UFSC (1997)

3. Carvalho, B. M. ; "Estudos de Cinética de Cristalização Aplicados ao Processo de Moldagem por Injeção"; Qualificação para doutoramento; DEMa-UFSCar (1995).

4. Kantz, M. R.; Newman, H. D.; Stiagle, F. H.; J. Appl. Polym. Sci.;v.16; p.1249 (1972).

5. Fujiyama, M.; Wakino, T.; J. Appl. Polym. Sci.; v.43; p.57 (1991).

6. Lafleur, P. G.; Kamal, M. R.; Polym. Eng. Sci.; v.26; p.92 (1986).

7. Kamal; M. R.; Lafleur, P. G.; Polym. Eng. Sci.; v.26; p.103 (1986).
8. Brito,A. C. G. “Simulação do Escoamento de Termoplásticos em Placas Moldadas por Injeção"; Tese de Doutorado; Universidade do Minho, Portugal (1995).

9. Brito, A. M.; Cunha, A. M.; Pouzada, A. S.; Crawford, R. J.; Int. Polym. Proc.; VI; p.370 (1991).

10. Hsiung, C. M.; Cakmak, M.; Polym. Eng. Sci.; v.31; p.1372 (1991).

11. Nakamura, K; Katayama, K.; Amano, T.; J. Appl. Polym. Sci.; v.17;p.1031 (1973).

12. Isayev, A. I.; Chan, T. W.; Shimojo, K.; Gmerek, M.; J. Appl. Polym. Sci.; v.55; p.807 (1995).

13. Isayev, A. I.; Chan, T. W.; Shimojo,K.; Gmerek, M.; J. Appl. Polym. Sci.; v.55; p.821 (1995).

14. Hoffman, J. D.; Davis, G. T., Lauritzen, J. I. "Treatise on Solid State Chemistry"; editado por N. B. Hannay; vol.3; cap.7; Plenum Press; New York; (1976).

15. Chan, T. W.; Isayev, A. I.; Polym. Eng. and Sci.; v.34; p.461 (1994).

16. Eder, G.; Janeschitz-Kriegl, H.; Liedauer, S.; Prog. Polym. Sci.; v.15; p.629 (1990).

17. Janeschitz-Kriegl, H.; Eder, G.; J. Macromol. Sci. Chem; A27; p.1733 (1990).

18. Sperling, L. H.; "Introduction to Physical Polymer Science"; 2.ed; John Wiley \& Sons; New York; (1992).

19. Patel, R. M.; Spruiell, J. E.; Polym. Eng. Sci.; v.31; p.730 (1991).

20 Sifleet, W. L.; Dinos, N.; Collier, J. R.; Polym. Eng. Sci.; v.13; p.10 (1973).

21. Godovsky, Y. K.; Slninsky, G. L.; J. Polym. Sci: Polym. Phys. Ed.; v.12; p.1053 (1974).

22. Bird R. B.; Armstrong, R. C.; Hassager, O. "Dynamics of Polymeric Liquids - Vol.1 Transport Phenomena"; John Wiley \& Sons; 2.ed (1987).

23. Cross, M. M.; Rheol. Acta, v.18, p.909 (1979).

24. Hieber, C. A. "Melt Viscosity Characterzation ans Its Application to Injection Molding"; em Injection and Compression Molding Fundamentals; cap. 1; ed. Avraam I. Isayev; Marcel Dekker, Inc.; New York (1987 ).

25. Patankar, S.; "Numerical Heat Transfer and Fluid Flow"; Hemisphere Publishing Corporation; Washington (1980).

26. Maliska, C. R.; "Transferência de Calor e Mecânica dos Fluidos Computacional"; LTC - Livros Técnicos e Científicos Editora S.A.; Rio de Janeiro (1995).

27. Kenedy. P.; "Flow Analysis of Injection Molds"; Hanser Publisher (1995). 